\title{
Does Processing or Formation of Water Ice Mantles Affect the Capacity of Nanosilicates to Be the Source of Anomalous Microwave Emission?
}

\author{
Joan Mariñoso Guiu ${ }^{1}$, Stefano Ferrero ${ }^{2}$, Antonio Macià Escatllar ${ }^{1}$, Albert Rimola ${ }^{2 \star}$ and \\ Stefan T. Bromley ${ }^{1,3 *}$
}

${ }^{1}$ Departament de Ciencia de Materials i Química Física \& Institut de Química Teòrica i Computacional (IQTCUB), Universitat de Barcelona, Barcelona, Spain, ${ }^{2}$ Departament de Química, Universitat Autònoma de Barcelona, Bellaterra, Spain, ${ }^{3}$ Institució Catalana de Recerca i Estudis Avançats (ICREA), Barcelona, Spain

\section{OPEN ACCESS}

Edited by: Luca Bizzocchi,

Max Planck Institute for Extraterrestrial

Physics, Germany

Reviewed by:

Martin Robert Stewart McCoustra Heriot-Watt University,

United Kingdom

Sergio loppolo,

Queen Mary University of London, United Kingdom

*Correspondence: Albert Rimola albert.rimola@uab.cat

Stefan T. Bromley

s.bromley@ub.edu

Specialty section: This article was submitted to Astrochemistry,

a section of the journal

Frontiers in Astronomy and Space

Sciences

Received: 05 March 2021 Accepted: 30 April 2021 Published: 20 May 2021

Citation:

Mariñoso Guiu J, Ferrero S,

Macià Escatllar A, Rimola $A$ and Bromley ST (2021) Does Processing or Formation of Water Ice Mantles Affect the Capacity of Nanosilicates to Be the

Source of Anomalous Microwave

Emission?

Front. Astron. Space Sci. 8:676548. doi: 10.3389/fspas.2021.676548
Anomalous microwave emission (AME) is detected in many astrophysical environments as a foreground feature typically peaking between $20-30 \mathrm{GHz}$ and extending over a $10-60 \mathrm{GHz}$ range. One of the leading candidates for the source of AME is small spinning dust grains. Such grains should be very small (approx. $\leq 1 \mathrm{~nm}$ diameter) in order for the rotational emission to fall within the observed frequency range. In addition, these nanosized grains should possess a significant dipole moment to account for the observed emissivities. These constraints have been shown to be compatible with spinning bare nanosilicate clusters, assuming that $\sim 1 \%$ of the total Si mass budget is held in these ultrasmall grains. Silicate dust can be hydroxylated by processing in the interstellar medium and is generally known to provide seeds for molecular water ice nucleation in denser regions. Herein, we use quantum chemical calculations to investigate how the dipole moment of $\mathrm{Mg}$-rich pyroxenic $\left(\mathrm{MgSiO}_{3}\right)$ nanoclusters is affected by both accretion of molecular water and dissociative hydration. Our work thus provides an indication of how the formation of water ice mantles is likely to affect the capacity of nanosilicates to generate AME.

Keywords: anomalous microwave emission, dust grains, nanosilicates, water ices, interstellar medium, grain processing, hydroxylation, density functional theory

\section{INTRODUCTION}

Silicate dust is ubiquitously found in a wide range of astrophysical environments (Henning, 2010). Chemical nucleation and growth in the circumstellar envelopes of oxygen-rich evolved stars is an important source of silicate dust (Goumans and Bromley, 2012; Gobrecht et al., 2016). From infrared (IR) emission observations, it is inferred that most of these newly formed silicate grains are Mg-rich and have sizes of the order $0.1 \mu \mathrm{m}$ (diameter) (Norris et al., 2012). Interactions between the dust and the stellar radiation field cause the grains to be ejected into the interstellar medium (ISM). Supernova shocks in the ISM induce grain-grain collisions which shatter the dust into smaller components (Jones et al., 1996). A typical shock is thought to result in 5-15\% of the original grain mass ending up in the form of ultrasmall nanosilicates $(<3 \mathrm{~nm}$ diameter). Nanograin production from larger grains is also predicted to occur close to sources of intense radiation (e.g., massive stars and supernovae) via rotational disruption of dust grains by radiative torques (Hoang et al., 2019). These models of dust 

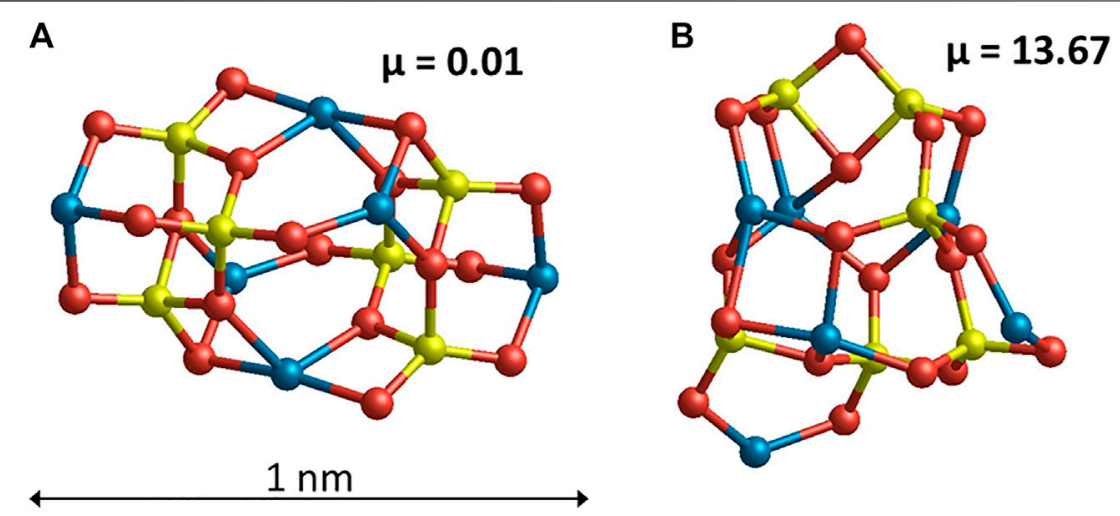

FIGURE 1 | Bare $\left(\mathrm{MgSiO}_{3}\right)_{6}$ nanosilicate structures: (A) low dipole (global minimum structure) and (B) high dipole (+1.11 eV relative to the global minimum). Dipole moments for each nanocluster are given in Debyes. Atom color key: silicon-yellow, magnesium-blue, and oxygen-red.
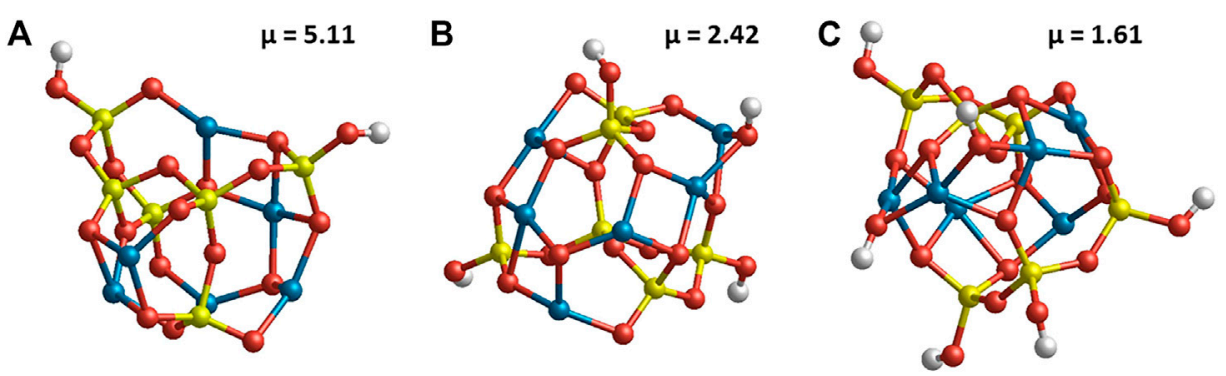

FIGURE 2 | Lowest energy hydroxylated $\left(\mathrm{MgSiO}_{3}\right)_{6}$ nanosilicate structures found with (A) one, (B) two, and (C) three water molecules. Dipole moments for each nanocluster are given in Debyes. Atom color key: silicon-yellow, magnesium-blue, and oxygen-red.

processing are compatible with estimates that approximately $10 \%$ of the $\mathrm{Si}$ in the ISM could be held in $\leq 3 \mathrm{~nm}$ diameter nanosilicate grains (Li and Draine, 2001) without violating observational constraints.

Via gas-grain collisions and interactions with radiation, small nanosilicate grains will be rotationally excited. Such spinning nanosilicate grains have been proposed as the main carrier of anomalous microwave emission (AME) - a microwave foreground feature which typically peaks between $20-30 \mathrm{GHz}$ and extends over a $10-60 \mathrm{GHz}$ range (Macià Escatllar and Bromley, 2020; Hoang et al., 2016; Hensley and Draine, 2017). AME is observed in a number of astrophysical environments including molecular clouds (Watson et al., 2005) and the diffuse ISM (Hildebrandt et al., 2007). As smaller grains can be spun up to larger frequencies than larger grains in any given astronomical environment, proportionally they emit more power, and thus the smallest grains are expected to dominate the spinning dust microwave spectrum. For a grain to emit in the microwave region associated with $\mathrm{AME}$ via rotational emission, its size should be of the order of $\sim 1 \mathrm{~nm}$ in diameter (Draine and Lazarian, 1998a; Draine and Lazarian, 1998b). In principle, contributions to the AME from the spinning of such nanograins could occur from any type of nanograin if it is sufficiently abundant and has a large enough dipole moment
(Ali-Haïmoud, 2013; Dickinson et al., 2018). Observational evidence has tended to rule out small spinning organic dust species (i.e., polycyclic aromatic hydrocarbons-PAHs) as the most important source of AME (Hensley et al., 2016). The atomistically detailed structures and physical properties of nanosilicates with $\mathrm{Mg}$-rich olivinic $\left(\mathrm{Mg}_{2} \mathrm{SiO}_{4}\right)_{\mathrm{N}}$ and pyroxenic $\left(\mathrm{MgSiO}_{3}\right)_{\mathrm{N}}$ compositions with diameters $\leq 1 \mathrm{~nm}$ have recently been determined by quantum chemical modeling (Macià Escatllar et al., 2019). These accurate calculations clearly show how the ionic chemical bonding in nanosilicates intrinsically tends to lead to grains with significant dipoles. Based on those structures, it has been shown that the AME can be explained if only $1 \%$ of the Si in the ISM were held in ultrasmall (i.e., $\leq 1 \mathrm{~nm}$ diameter) nanosilicate grains (Macià Escatllar and Bromley, 2020). Although the dipoles possessed by these bare nanosilicates are more than sufficient to account for the observed AME, it is not clear if these results can be extended to nanosilicates, which become hydroxylated by processing and/ or upon which water ice forms.

Silicate grains emerging from the outflows of evolved stars are processed from ion irradiation (e.g., protons, $\mathrm{He}^{+}$) from supernovae shocks in the ISM. From laboratory studies of the effect of the solar wind on lunar surface silicate material, proton irradiation was found to cause two important intertwined 

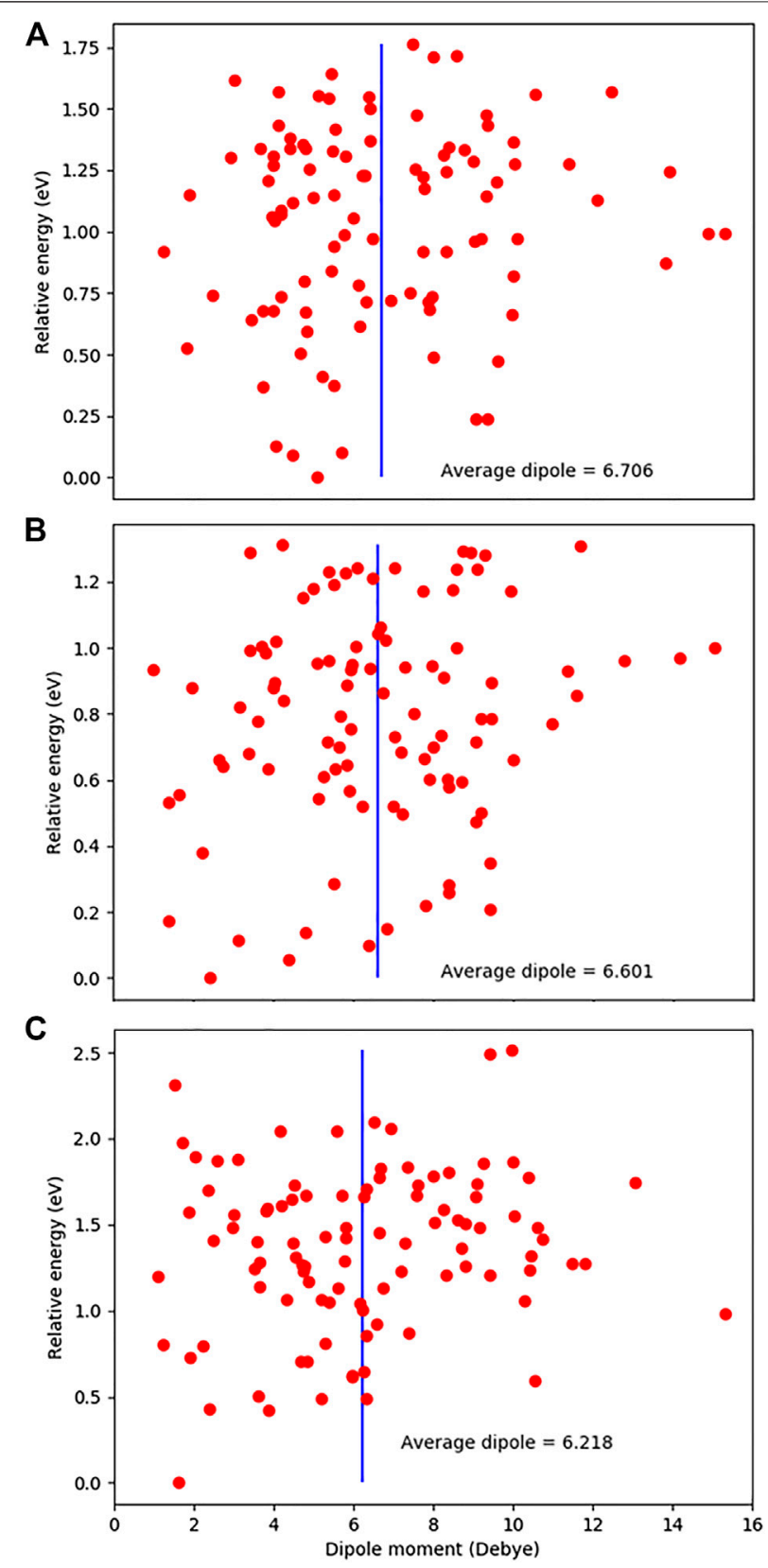

FIGURE 3 | Plot of relative energy vs. dipole moment for hydroxylated $\left(\mathrm{MgSiO}_{3}\right)_{6}$ nanosilicates for $(\mathbf{A})$ one, (B) two, and (C) three water molecules. The vertical blue line denotes the average dipole moment of the set.

chemical processes: 1) hydroxyl formation (Zeller et al., 1966) and 2) $\mathrm{Mg}$ cation depletion by preferential sputtering (Keller and Mckay, 1997). Analysis of interplanetary dust has provided direct evidence of such grain processing (Bradley, 1994). Such processes would tend to transform $\mathrm{Mg}$-rich nano-olivine dust grains into hydroxylated nano-pyroxene grains. Relatively elevated levels of pyroxene (with respect to the initial dominance of olivine) are consistent with the pyroxene-rich environments of protostars (Demyk et al., 1999) and the estimated fraction of amorphous pyroxene grains in the ISM (Kemper et al., 2004).
In denser regions of the ISM such as molecular clouds, the ultraviolet radiation field is shielded and molecular water can accrete on silicate grain surfaces. This marks the beginning of the formation of water ice-dominated mantles on silicate grains. Water is one of the most abundant molecules in the universe in the solid phase (Gibb et al., 2004; Boogert et al., 2008). Interstellar water ices are thought to present an amorphous structural state because of the similarities of their IR features with those of amorphous solid water measured in terrestrial laboratories (Watanabe and Kouchi, 2008). Gas-phase reactions based on low-temperature ion-neutral and high-temperature neutralneutral processes allow for the explaining of the abundance of water as a gaseous component, but they cannot justify the large abundance of water ice in interstellar and protostellar clouds (Hollenbach and McKee, 1989; van Dishoeck et al., 2013). Accordingly, reaction routes based on surface chemical processes have long been recognized to be the major channel for the formation of water ice (Tielens and Hagen, 1982; van Dishoeck et al., 2013). Therefore, water ice, rather than being formed by successive adsorption of water molecules coming from the gas phase, is formed in situ on the surfaces of dust grains. Different reactive channels leading to the formation of interstellar water on the surfaces of grains are hydrogenation of atomic $\mathrm{O}$ (e.g., Dulieu et al., 2010), of molecular $\mathrm{O}_{2}$ (e.g., Ioppolo et al., 2008), and even of $\mathrm{O}_{3}$ (e.g., Mokrane et al., 2009), among others. We refer to the work of Molpeceres et al. (2019) for a complete list of postulated reactions leading to water formation on interstellar grains.

Herein, we perform a systematic quantum chemical study of the effect of both hydroxylation and molecular water interactions on ultrasmall ( $\sim 1 \mathrm{~nm}$ diameter) Mg-rich pyroxenic nanosilicates with a $\left(\mathrm{MgSiO}_{3}\right)_{6}$ composition. We specifically focus on how the dipole moments of the original bare P-6 nanosilicates are affected by these chemical changes. If we focus on the dipole moments of the most energetically stable grains in a population, we find that both processes can significantly affect the dipole moment of these selected grains. However, when considering a more realistic population of nanosilicate grains, our results suggest that neither hydroxylation nor molecular hydration has a significant effect on the average magnitude of the dipole moment. Our results thus suggest that the capacity of nanosilicates to be the source of AME should not be significantly diminished by processing and/or initial water ice mantle formation.

\section{METHODS}

The initial bare $\left(\mathrm{MgSiO}_{3}\right)_{6}$ isomer structures were obtained in a previous work (Macià Escatllar et al., 2019). We chose to compare two distinct isomers: 1) the lowest energy (global minimum) cluster structure with a very low dipole and 2) a slightly energetically metastable cluster structure with a high dipole. These bare $\left(\mathrm{MgSiO}_{3}\right)_{6}$ structures are shown in Figure 1 .

In the case of hydroxylated bare nanosilicate clusters, we carried out global optimization calculations to find the 50 lower energy isomers of $\left(\mathrm{MgSiO}_{3}\right)_{6}$ after dissociative 
TABLE 1 | Evolution of total dipole moments $(\mu)$ in Debyes of $\left(\mathrm{MgSiO}_{3}\right)_{6}$ nanosilicates with increasing hydration via hydroxylation (chemisorption) and molecular water adsorption (physisorption). The average dipoles of the hydrated nanosilicates are based on the data obtained herein and shown in Figures $\mathbf{2 - 4}$. Values of $\beta$ are calculated as $\beta=\mu / \sqrt{ } N_{\text {atoms }}$.

\begin{tabular}{|c|c|c|c|c|}
\hline Water addition process & $\begin{array}{c}\text { Dipole moment of } \\
\text { initial bare nanosilicates } / \beta\end{array}$ & $\begin{array}{l}\text { Average dipole moment } / \beta \\
\qquad\left(+1 \times \mathrm{H}_{2} \mathrm{O}\right)\end{array}$ & $\begin{array}{l}\text { Average dipole moment } / \beta \\
\qquad\left(+2 \times \mathrm{H}_{2} \mathrm{O}\right)\end{array}$ & $\begin{array}{l}\text { Average dipole moment } / \beta \\
\qquad\left(+3 \times \mathrm{H}_{2} \mathrm{O}\right)\end{array}$ \\
\hline Hydroxylation (chemisorption) & $7.7^{\mathrm{a} / 1.41}$ & $6.71 / 1.17$ & $6.60 / 1.1$ & $6.22 / 1.04$ \\
\hline Molecular adsorption (physisorption) & $0.01^{b} / 0.002$ & $2.87 / 0.50$ & $3.32 / 0.55$ & $3.30 / 0.55$ \\
\hline Molecular adsorption (physisorption) & $13.67^{\mathrm{c}} / 2.50$ & $14.40 / 2.51$ & $11.97 / 2.00$ & $12.65 / 2.12$ \\
\hline
\end{tabular}

${ }^{a}$ Average value of dipole moment calculated from a set of $180\left(\mathrm{MgSiO}_{3}\right)_{6}$ structures-see Macià Escatllar and Bromley (2020).

${ }^{b}$ Dipole moment of low dipole global minimum $\left(\mathrm{MgSiO}_{3}\right)_{6}$ structure-see Macià Escatllar et al. (2019) and Figure 1A.

${ }^{c}$ Dipole moment of high dipole $\left(\mathrm{MgSiO}_{3}\right)_{6}$ structure $(+1.1 \mathrm{eV}$ with respect to the global minimum) - see Figure $1 \mathbf{B}$.

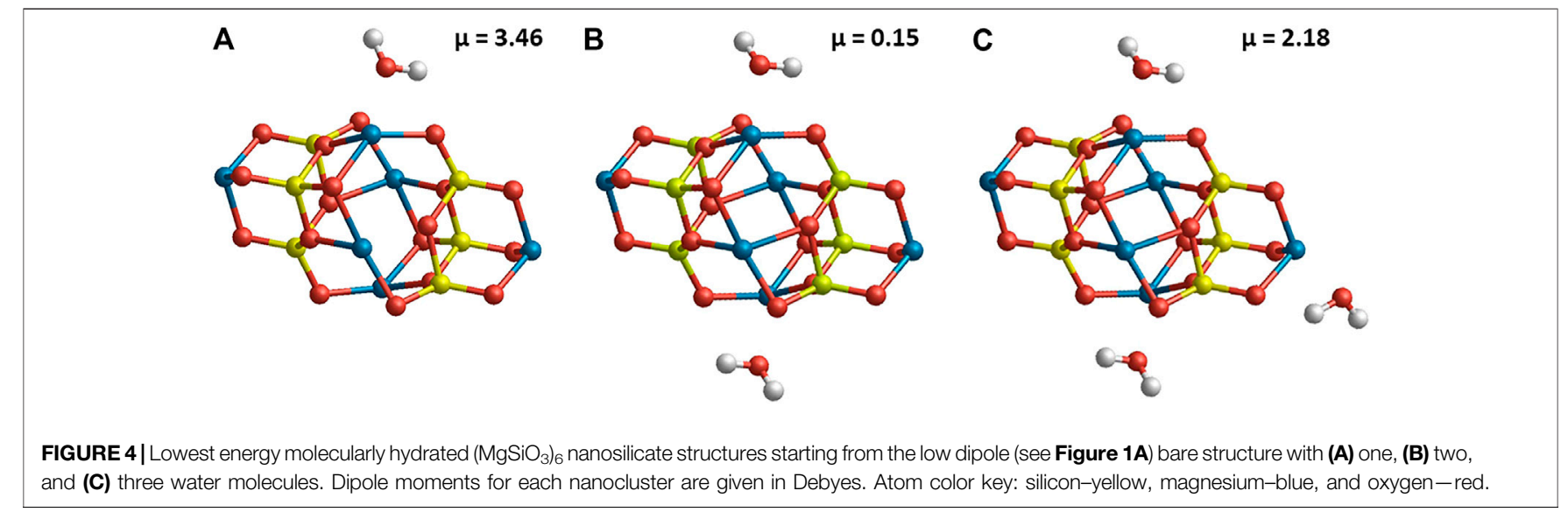

interaction of one, two, and three water molecules, respectively. The global optimization calculations were split into two steps. First, we used the Monte Carlo basin hopping (MCBH) algorithm (Wales and Doye, 1997), employing previously developed polarizable classical interatomic potentials (IPs) (Macià Escatllar et al., 2019), to explore the potential energy surface (PES), followed by the structural configurations of the hydroxylated clusters. Previous studies have shown that the $\mathrm{MCBH}$ method is an efficient method for exploring the low energy PES of nanoclusters and for finding the global minimum (Wales and Doye, 1997). The standard MCBH algorithm uses random atomic displacements at each step to explore the PES. In addition, we also allowed that $0.5 \%$ of the steps attempt an $\mathrm{Mg} \leftrightarrow \mathrm{Si}$ cation exchange move assist in this exploration. To run the MCBH calculations, we employed a previously developed cascade MCBH code (Cuko et al., 2017), which uses the General Utility Lattice Package (GULP) (Gale and Rohl, 2003) to locally optimize the cluster structures at each step. To avoid problems with the local optimization of distorted structures containing polarized ions, we preoptimize each cluster structure with an IP with polarizable shells removed before optimizing using the IP with shells. We note that, in principle, an MCBH global optimization search only depends on the chemical composition of the system and should be independent of the initial hydroxylated cluster structure. In practice, the exploration of the PES can be somewhat biased by the initial structure and thus, to help ensure that our searches were not biased, for each addition of water, we ran the MCBH searches a total of 20 times with different starting structures. Each $\mathrm{MCBH}$ run consisted of 10,000 steps.

After this global optimization procedure, we selected 100 distinct lowest energy nanocluster structures for each degree of hydroxylation for further refinement. The selected cluster structures were optimized using density functional theory (DFT)-based calculations using the PBE functional (Perdew et al., 1996) with a light-tier one numerical atom-centered orbital (NAO) basis set. Previous studies on oxide nanoparticles have shown that this basis set has a similar quality to valence triple- $\zeta$ plus polarization Gaussian-type Orbital basis sets (Lamiel-Garcia et al., 2017). These DFTbased calculations employed the Fritz Haber Institute $\mathrm{Ab}$ Initio Molecular Simulation package (FHI-AIMS) (Blum et al., 2009).

In the case of the molecularly hydrated $\left(\mathrm{MgSiO}_{3}\right)_{6}$ clusters, the initial positions of the physisorbed water molecules on both bare cluster isomers (Figure 1) were prepared using an electrostatic compatibility criterion. Specifically, the negatively charged oxygen atom of each water molecule was placed at a distance $2 \AA$ above each positively charged magnesium cation of the silicate cluster. Subsequently, for each water + cluster combination, the whole system structure was locally optimized. All optimizations were carried out using DFTbased calculations using the PBE functional and a def2- 

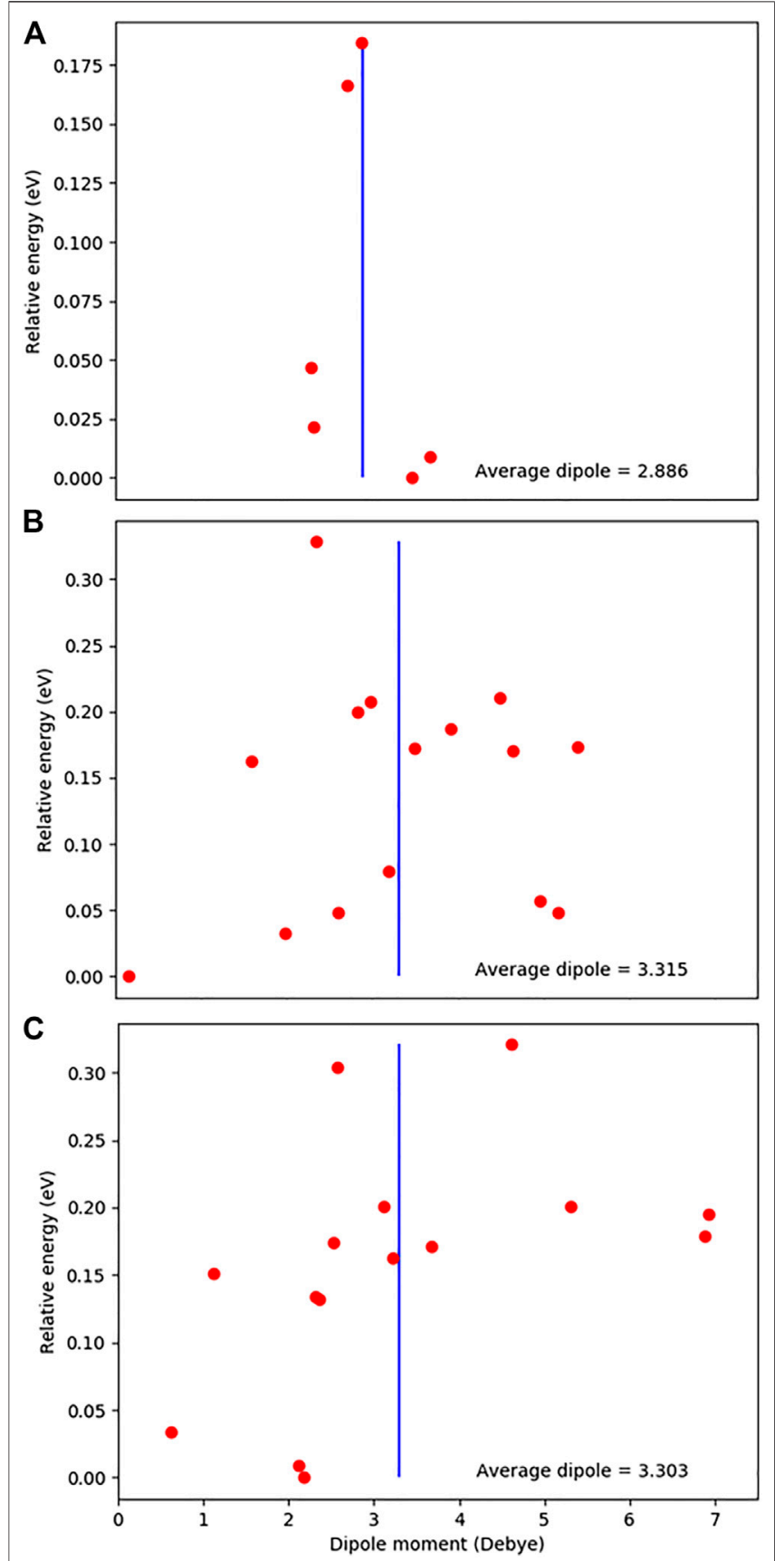

FIGURE 5 | Plot of relative energy vs. dipole moment for molecularly hydrated $\left(\mathrm{MgSiO}_{3}\right)_{6}$ nanosilicates based on the low dipole bare nanosilicate (Figure 1A) for (A) one, (B) two, and (C) three water molecules. The vertical blue line denotes the average dipole moment of each set. In each plot, data from approximately 100 isomers are shown.

TZVPP (i.e., valence triple- $\zeta$ plus polarization) basis set (Weigend and Ahlrichs, 2005). This process was repeated for sampling all the $\mathrm{Mg}$ sites and all distinct combinations of adsorption sites when using two and three water molecules. The ORCA code (Neese et al., 2020) was used for these calculations.

\section{RESULTS AND DISCUSSION}

In the following subsections, we describe our findings for both hydroxylation and molecular interaction with water for bare $\left(\mathrm{MgSiO}_{3}\right)_{6}$ nanosilicate clusters (see Figure 1). Note that these bare nanosilicates contain 30 atoms and have a typical maximum diameter of $\sim 1 \mathrm{~nm}$. The hydrated $\left(\mathrm{MgSiO}_{3}\right)_{6}\left(\mathrm{H}_{2} \mathrm{O}\right)_{n}$ nanosilicates we consider have up to 39 atoms, which induces only a minor increase in the average diameter. Although the chemical compositions of the hydrated nanosilicate systems considered in each case (i.e., hydroxylation and molecular interactions) are identical, astronomically each relate to distinct processes and regions of the ISM. As such, we discuss the two considered modes of nanosilicate hydration separately with relation to their astronomical context.

\section{Dissociative Hydration}

As discussed above, hydroxylation of silicate dust can occur through high energy processing by supernovae shocks in the diffuse ISM. Hydroxylation is also the natural chemical result of dissociative chemisorption of water molecules on dust grain surfaces. However, as water molecules are quickly photodissociated by high-energy ultraviolet (UV) photons in the diffuse ISM, chemical routes to hydroxylated nanosilicate dust would more likely occur via reactions with other species (e.g., $\mathrm{O}$ and $\mathrm{H}$ atoms). We note that the explicit modeling of these reactions and how the resulting hydroxylation affects subsequent $\mathrm{H}_{2}$ formation reactions were investigated in previous works on a $\left(\mathrm{MgSiO}_{3}\right)_{4}$ nanopyroxene cluster (Goumans and Bromley, 2011; Kerkeni et al., 2017; Kerkeni et al., 2019). Generally, hydroxylation of silicate grain surfaces is the most plausible mechanism by which hydration of silicates can occur in the diffuse ISM.

We consider hydroxylated nanopyroxene clusters with chemical compositions $\left(\mathrm{MgSiO}_{3}\right)_{6}\left(\mathrm{H}_{2} \mathrm{O}\right)_{n}$, with $n=1-3$. After finding the low-energy minima of these systems using the $\mathrm{MCBH}$ global optimization method, although some of the higher energy isomers displayed physisorbed water molecules, most clusters exhibited $2 n$ hydroxyls. First, this shows that hydroxylation is energetically more favorable than molecular water adsorption. The lowest energy (i.e., candidate global minima) hydroxylated isomers found for each $n$ are shown in Figure 2. By only considering the lowest energy isomers, we can follow the evolution of the dipole moment from the bare $\left(\mathrm{MgSiO}_{3}\right)_{6}$ with increasing hydroxylation (see Figure 2). Second, we note that the global minimum bare $\left(\mathrm{MgSiO}_{3}\right)_{6}$ nanosilicate isomer has a negligible dipole moment, due to its high symmetry. However, all lowest energy hydroxylated nanosilicates have a significant dipole moment and correspondingly low symmetry structures. Based on this relatively small change in the degree of hydroxylation for the lowest energy isomers, the initial relatively large increase in the dipole moment going from a bare cluster to one with two hydroxyls (+5.1 Debye) is then dampened with further hydroxylation increase.

In the ISM, due to energetic processing, the population of nanosilicates is likely to exhibit a range of isomeric structures and compositions with a corresponding range of energetic stabilities. 

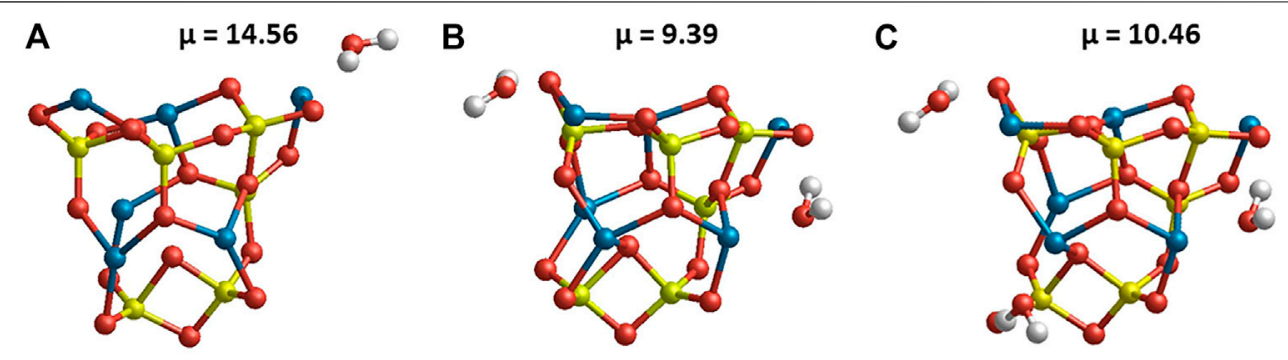

FIGURE 6 | Lowest energy molecularly hydrated $\left(\mathrm{MgSiO}_{3}\right)_{6}$ nanosilicate structures starting from the high dipole (see Figure 1B) bare structure with (A) one, (B) two, and (C) three water molecules. Dipole moments for each nanocluster are given in Debyes. Atom color key: silicon-yellow, magnesium-blue, and oxygen-red.

From an astronomical perspective, it is thus more relevant to extract average properties of grain populations. From this more general perspective, it has previously been shown that the high symmetry of the global minimum bare $\left(\mathrm{MgSiO}_{3}\right)_{6}$ nanosilicate is not typical of the majority of isomers. In fact, the average dipole moment of $\left(\mathrm{MgSiO}_{3}\right)_{6}$ isomers, considering over 100 lowest energy structures, is found to be 7.7 Debyes (Macià Escatllar and Bromley, 2020). In the case of the hydroxylated nanosilicates considered herein, our global optimization searches also produce a large number of low-energy isomer structures from which to obtain the average dipole moments. In Figure 3, we show the relative energetic stability and corresponding dipole moments for approximately 100 distinct nanocluster isomers for each degree of hydroxylation (i.e., $n=1-3$ ) considered. In Table 1, we report the average dipole moments of all hydroxylated $\left(\mathrm{MgSiO}_{3}\right)_{6}\left(\mathrm{H}_{2} \mathrm{O}\right)_{n}$ nanoclusters based on these data. It has been shown that for a dipolar nanocluster species to contribute to AME, it should possess a sufficiently large size-normalized dipole moment $\left(\beta=\mu / \sqrt{ } N_{\text {atoms }}\right)$ such that $\beta>0.3$ (Hoang et al., 2016, Hensley and Draine, 2017). In Table 1, we also reported values of $\beta$ based on the average dipole moments on our sets of hydroxylated nanosilicate clusters. We note that although there is a very slight decrease in the average dipole moment with increasing hydroxylation, the value of $\beta$ is always over three times the size required according to the above AME constraint. These data clearly show that in all cases, a population of hydroxylated $\left(\mathrm{MgSiO}_{3}\right)_{6}\left(\mathrm{H}_{2} \mathrm{O}\right)_{n}$ nanoclusters could significantly contribute to the observed AME.

\section{Molecular Hydration}

Hydroxylation via shock-induced processing or suitable chemical reactions is initially very energetically favorable for bare grains, but, with increasing hydroxylation, the surface sites for the hydroxylation to take place quickly become occupied. As such, nanosized grains can only maintain a limited number of hydroxyls on their surfaces before they become too energetically favorable for any formed water to interact with the hydroxylated grain in a molecular physisorbed manner (Kerkeni and Bromley, 2013). As mentioned above, the main mechanism through which water ice forms and grows on the core grains (e.g., silicates) is by cumulative water formation on the same grain surface, where it remains firmly attached. Here, to check how the presence of interacting water molecules affects the dipole moment of the bare nanograin, we assume that water formation has already taken place on the binding sites where the water molecules are molecularly adsorbed.
Following our results for hydroxylation, we also consider the initial stages of molecular hydration in $\left(\mathrm{MgSiO}_{3}\right)_{6}\left(\mathrm{H}_{2} \mathrm{O}\right)_{n}$ species for $n=1-3$. Note that unlike in the energetic process of hydroxylation in the ISM, in protected molecular cloud environments, each molecular hydration is a low-energy event in which we assume that the bare nanocluster structure is unaffected. We take the two nanocluster structures in Figure 1 as seeds for studying the initial stages of molecular physisorption. First, we consider the global minimum low dipole nanocluster (see Figure 1A) which has a negligible dipole due to its very symmetric structure. In Figure 4, we show the lowest energy $\left(\mathrm{MgSiO}_{3}\right)_{6}\left(\mathrm{H}_{2} \mathrm{O}\right)_{n}$ structures that we obtained for the addition of 1-3 water molecules. Upon nucleation of the first water molecule, the symmetry of the cluster is broken, resulting in a dramatic increase in the dipole moment. Conversely, with two water molecules, the lowest energy structure is highly symmetric, leading to a very low dipole moment. Finally, with three water molecules, the symmetry is once again broken and the lowest energy system is found to exhibit a high dipole moment. As in the case of the hydroxylated clusters, in astronomical environments there is likely to be a range of isomers for each degree of hydration, and the average effect of molecular hydration is of more interest. In this case, due to the limited number of distinct binding sites, we have fewer possibilities for each water addition than in the case of hydroxylated nanocluster isomers. Specifically, for the nucleation of one water molecule, we find six distinct adsorption sites, and for two and three water molecules, we consider 15 distinct adsorption sites. The relative energies and dipole moments of all these systems are shown in Figure 5. The corresponding average dipole moments (see Table 1) are all close to 3 Debyes, showing that molecular water adsorption can induce an average increase in the dipole moment, even for bare nanoclusters with negligible dipole moments. The corresponding values of $\beta$ are all close to 0.5 , which is more than sufficient for such hydrated nanoclusters to contribute to AME.

In the case of molecular nucleation of a bare cluster with an initial large dipole moment (see Figure 1B), we show the lowest energy $\left(\mathrm{MgSiO}_{3}\right)_{6}\left(\mathrm{H}_{2} \mathrm{O}\right)_{\mathrm{n}}$ structures that we obtained in our searches in Figure 6. For these specific low-energy structures, we see that the dipole moment stays high ( $>9$ Debyes) for each water addition. More generally, we consider a similar range of hydrated structures as in the low dipole moment case to obtain averages. In Figure 7, we show the relative energies and dipole moments of all considered systems. Here, we also see that the 

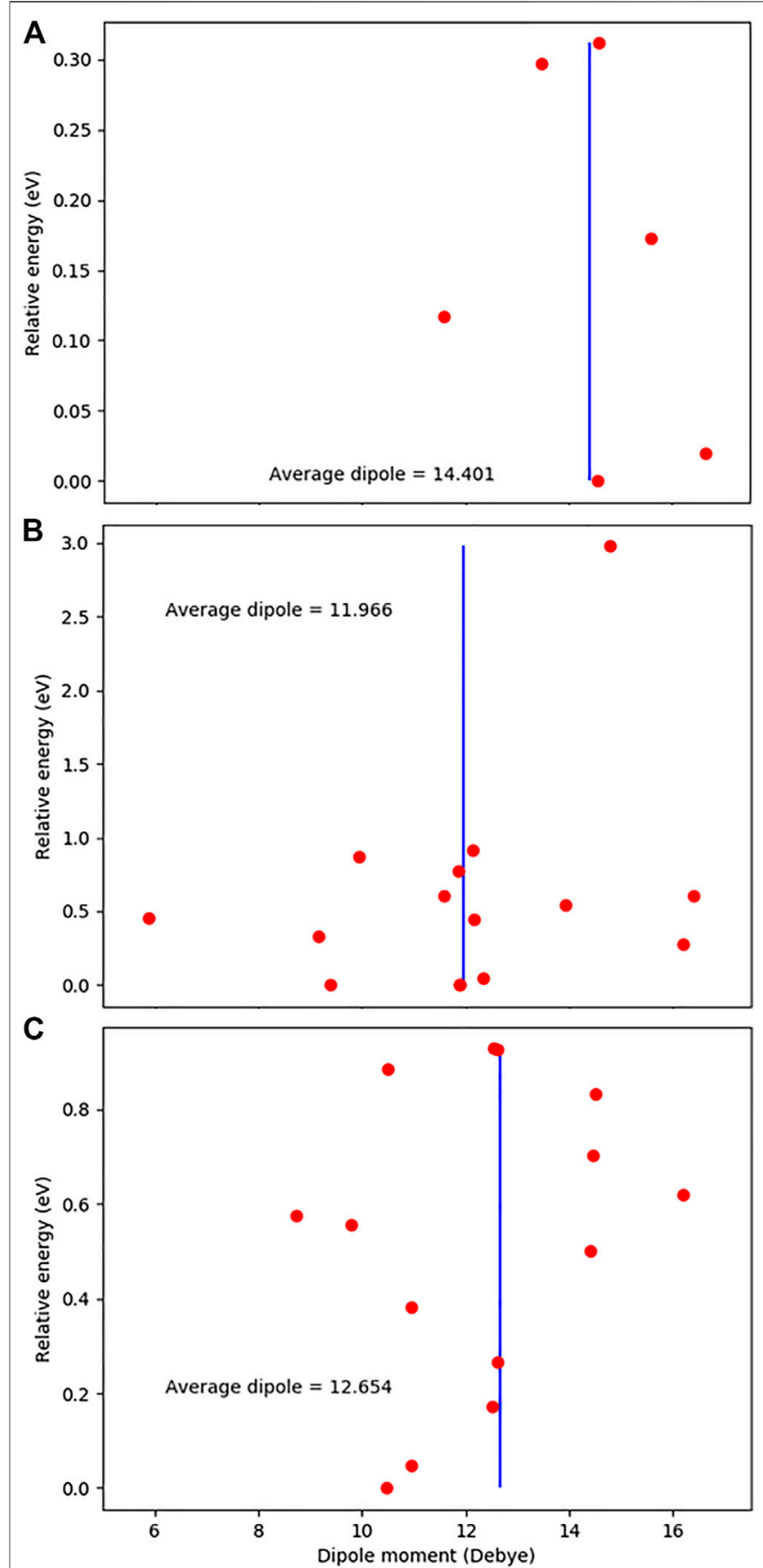

FIGURE 7 | Plot of relative energy vs. dipole moment for molecularly hydrated $\left(\mathrm{MgSiO}_{3}\right)_{6}$ nanosilicates based on the high dipole bare nanosilicate (Figure 1B) for (A) one, (B) two, and (C) three water molecules. The vertical blue line denotes the average dipole moment of each set. average dipole moments (see also Table 1) for each degree of hydration also stay high. Correspondingly, the values of $\beta$ obtained from these average dipole moment values are also high ( $>2$ Debyes), meaning that they would be strong emitters of microwave radiation.

\section{CONCLUSION}

We use searches based on global optimization and electrostatic considerations to obtain a range of structures for hydroxylated and molecularly hydrated nanoclusters, respectively, with a $\left(\mathrm{MgSiO}_{3}\right)_{6}\left(\mathrm{H}_{2} \mathrm{O}\right)_{n}$ composition $(n=1-3)$. These hydrated nanopyroxenes could be relevant for nanosilicate grain processing in the ISM or initial water ice mantle formation in molecular clouds. We have used accurate quantum chemical DFT calculations to calculate the dipole moments of all hydrated pyroxene nanoclusters. We find that both hydroxylation and molecular hydration, irrespective of the initial dipole moment of the bare nanocluster, induce and/or maintain systems with dipole moments that are sufficiently high to significantly contribute to AME. These results thus strongly indicate that the validity of previous reports showing that nanosilicates are very plausible carriers of AME is not affected by a modest degree of nanosilicate hydration. Overall, this study broadens the range of potential nanosilicate species and types of astrophysical environments that could produce AME signatures.

\section{DATA AVAILABILITY STATEMENT}

The data supporting the findings of this study are available on reasonable request from the corresponding authors.

\section{AUTHOR CONTRIBUTIONS}

The original idea for the study was proposed by SB. All authors participated in the initial conception and planning of the study and in the discussion of the results. JMG and SF performed the calculations. SB and AR participated in the writing and editing of the manuscript and coordinated the project.

\section{FUNDING}

This project has received funding from the European Union's Horizon 2020 research and innovation program under the Marie Sklodowska-Curie grant agreement No. 811312 for the project "AstroChemical Origins" (ACO) and from the European Research Council (ERC) under the European Union's Horizon 2020 research and innovation program grant agreement No. 865657 for the project "Quantum Chemistry on Interstellar Grains" (QUANTUMGRAIN). We acknowledge financial support from the Spanish Ministerio de Ciencia, Innovación y Universidades (projects CTQ 2017-89132-P, RTI 2018-095460-B-100, and MDM-2017-0767 via the Spanish Structures of Excellence María de Maeztu program) and the Generalitat de Catalunya (projects 2017SGR1323 and 2017SGR13).

\section{ACKNOWLEDGMENTS}

AR is indebted to the "Ramón y Cajal" program. The Red Española de Supercomputación (RES) and Consorci de Serveis Universitaris de Catalunya (CSUC) are also acknowledged for the provision of supercomputing time. 


\section{REFERENCES}

Ali-Haïmoud, Y. (2013). Spinning Dust Radiation: A Review of the Theory. Adv. Astron. 2013, 462697. doi:10.1155/2013/462697

Blum, V., Gehrke, R., Hanke, F., Havu, P., Havu, V., Ren, X., et al. (2009). Ab Initio Molecular Simulations with Numeric Atom-Centered Orbitals. Comput. Phys. Commun. 180, 2175-2196. doi:10.1016/j.cpc.2009.06.022

Boogert, A. C. A., Pontoppidan, K. M., Knez, C., Lahuis, F., Kessler-Silacci, J., van Dishoeck, E. F., et al. (2008). The c2dSpitzerSpectroscopic Survey of Ices Around Low-Mass Young Stellar Objects. I. H2O and the 5-8 $\mu \mathrm{m}$ Bands1,2. ApJ 678, 985-1004. doi:10.1086/533425

Bradley, J. P. (1994). Chemically Anomalous, Preaccretionally Irradiated Grains in Interplanetary Dust from Comets. Science 265, 925-929. doi:10.1126/science. 265.5174.925

Cuko, A., Macià, A., Calatayud, M., and Bromley, S. T. (2017). Global Optimisation of Hydroxylated Silica Clusters: A Cascade Monte Carlo Basin Hopping Approach. Comput. Theor. Chem. 1102, 38-43. doi:10. 1016/j.comptc.2016.12.030

Demyk, K., Jones, A. P., Dartois, E., Cox, P., and d'Hendecourt, L. (1999). The Chemical Composition of the Silicate Dust Around RAFGL7009S and IRAS 19110+1045. Astron. Astrophys. 349, 267-275.

Dickinson, C., Ali-Haïmoud, Y., Barr, A., Battistelli, E. S., Bell, A., Bernstein, L., et al. (2018). The State-Of-Play of Anomalous Microwave Emission (AME) Research. New Astron. Rev. 80, 1-28. doi:10.1016/j.newar.2018.02.001

Draine, B. T., and Lazarian, A. (1998a). Electric Dipole Radiation from Spinning Dust Grains. ApJ 508, 157-179. doi:10.1086/306387

Draine, B. T., and Lazarian, A. (1998b). Diffuse Galactic Emission from Spinning Dust Grains. Astrophys. J. 494, L19-L22. doi:10.1086/311167

Dulieu, F., Amiaud, L., Congiu, E., Fillion, J.-H., Matar, E., Momeni, A., et al. (2010). Experimental Evidence for Water Formation on Interstellar Dust Grains by Hydrogen and Oxygen Atoms. Astron. Astrophys. 512, A30. doi:10.1051/0004-6361/200912079

Gale, J. D., and Rohl, A. L. (2003). The General Utility Lattice Program (GULP). Mol. Simul. 29, 291-341. doi:10.1080/0892702031000104887

Gibb, E. L., Whittet, D. C. B., Boogert, A. C. A., and Tielens, A. G. G. M. (2004). Interstellar Ice: The Infrared Space Observatory Legacy. Astrophys J. Suppl. S 151, 35-73. doi:10.1086/381182

Gobrecht, D., Cherchneff, I., Sarangi, A., Bromley, S. T., and Bromley, S. T. (2016). Dust Formation in the Oxygen-Rich AGB Star Ik Tauri. A\&A 585, A6. doi:10. 1051/0004-6361/201425363

Goumans, T. P. M., and Bromley, S. T. (2011). Hydrogen and Oxygen Adsorption on a Nanosilicate - a Quantum Chemical Study. Mon. Not. R. Astron. Soc. 414, 1285-1291. doi:10.1111/j.1365-2966.2011.18463.x

Goumans, T. P. M., and Bromley, S. T. (2012). Efficient Nucleation of Stardust Silicates via Heteromolecular Homogeneous Condensation. Mon. Not. R. Astron. Soc. 420, 3344-3349. doi:10.1111/j.1365-2966. 2011.20255.x

Henning, T. (2010). Cosmic Silicates. Annu. Rev. Astron. Astrophys. 48, 21-46. doi:10.1146/annurev-astro-081309-130815

Hensley, B. S., and Draine, B. T. (2017). Modeling the Anomalous Microwave Emission with Spinning Nanoparticles: No PAHs Required. ApJ 836, 179. doi:10.3847/1538-4357/aa5c37

Hensley, B. S., Draine, B. T., and Meisner, A. M. (2016). A Case against Spinning Pahs as the Source of the Anomalous Microwave Emission. ApJ 827, 45. doi:10. 3847/0004-637x/827/1/45

Hildebrandt, S. R., Rebolo, R., Rubiño-Martín, J. A., Watson, R. A., Gutiérrez, C. M., Hoyland, R. J., et al. (2007). COSMOSOMAS Observations of the Cosmic Microwave Background and Galactic Foregrounds at $11 \mathrm{GHz}$ : Evidence for Anomalous Microwave Emission at High Galactic Latitude. Mon. Not. R. Astron. Soc. 382, 594-608. doi:10.1111/j.1365-2966.2007.12380.x

Hoang, T., Vinh, N.-A., and Lan, N. Q. (2016). Spinning Dust Emission from Ultrasmall Silicates: Emissivity and Polarization Spectrum. ApJ 824, 18. doi:10.3847/ 0004-637x/824/1/18

Hoang, T., Tram, L. N., Lee, H., and Ahn, S.-H. (2019). Rotational Disruption of Dust Grains by Radiative Torques in Strong Radiation Fields. Nat. Astron. 3, 766-775. doi:10.1038/s41550-019-0763-6
Hollenbach, D., and McKee, C. F. (1989). Molecule Formation and Infrared Emission in Fast Interstellar Shocks. III - Results for J Shocks in Molecular Clouds. ApJ 342, 306. doi:10.1086/167595

Ioppolo, S., Cuppen, H. M., Romanzin, C., van Dishoeck, E. F., and Linnartz, H. (2008). Laboratory Evidence for Efficient Water Formation in Interstellar Ices. Astrophys. J. 686, 1474-1479. doi:10.1086/591506

Jones, A. P., Tielens, A. G. G. M., and Hollenbach, D. J. (1996). Grain Shattering in Shocks: The Interstellar Grain Size Distribution. ApJ 469, 740. doi:10.1086/ 177823

Keller, L. P., and McKay, D. S. (1997). The Nature and Origin of Rims on Lunar Soil Grains. Geochim. Cosmochim. Acta 61, 2331-2341. doi:10.1016/s0016-7037(97) 00085-9

Kemper, F., Vriend, W. J., and Tielens, A. G. G. M. (2004). The Absence of Crystalline Silicates in the Diffuse Interstellar Medium. ApJ 609, 826-837. doi:10.1086/421339

Kerkeni, B., and Bromley, S. T. (2013). Competing Mechanisms of Catalytic H2 Formation and Dissociation on Ultrasmall Silicate Nanocluster Dust Grains. Mon. Not. R. Astron. Soc. 435, 1486-1492. doi:10.1093/mnras/stt1389

Kerkeni, B., Bacchus-Montabonel, M.-C., and Bromley, S. T. (2017). How Hydroxylation Affects Hydrogen Adsorption and Formation on Nanosilicates. Mol. Astrophys. 7, 1-8. doi:10.1016/j.molap.2017.04.001

Kerkeni, B., Bacchus-Montabonel, M.-C., Shan, X., and Bromley, S. T. (2019). Understanding H2 Formation on Hydroxylated Pyroxene Nanoclusters: Ab Initio Study of the Reaction Energetics and Kinetics. J. Phys. Chem. A. 123, 9282-9291. doi:10.1021/acs.jpca.9b06713

Lamiel-Garcia, O., Ko, K. C., Lee, J. Y., Bromley, S. T., and Illas, F. (2017). When Anatase Nanoparticles Become Bulklike: Properties of Realistic TiO2 Nanoparticles in the 1-6 Nm Size Range from All Electron Relativistic Density Functional Theory Based Calculations. J. Chem. Theor. Comput 13, 1785-1793. doi:10.1021/acs.jctc.7b00085

Li, A., and Draine, B. T. (2001). On Ultrasmall Silicate Grains in the Diffuse Interstellar Medium. Astrophys. J. Lett. 550, L213-L217. doi:10.1086/319640

Macià Escatllar, A., and Bromley, S. T. (2020). Assessing the Viability of Silicate Nanoclusters as Carriers of the Anomalous Microwave Emission: a Quantum Mechanical Study. Astronom. Astrophys. 634, A77. doi:10.1051/0004-6361/ 201936419

Macià Escatllar, A., Lazaukas, T., Woodley, S. M., and Bromley, S. T. (2019). Structure and Properties of Nanosilicates with Olivine (Mg2SiO4) N and Pyroxene (MgSiO3) N Compositions. ACS Earth Sp. Chem. 3, 2390-2403. doi:10.1021/acsearthspacechem.9b00139

Mokrane, H., Chaabouni, H., Accolla, M., Congiu, E., Dulieu, F., Chehrouri, M., et al. (2009). Experimental Evidence for Water Formation via Ozone Hydrogenation on Dust Grains at 10 K. ApJ 705, L195-L198. doi:10.1088/ 0004-637x/705/2/1195

Molpeceres, G., Rimola, A., Ceccarelli, C., Kästner, J., Ugliengo, P., and Maté, B. (2019). Silicate-mediated Interstellar Water Formation: A Theoretical Study. Mon. Not. R. Astron. Soc. 482, 5389-5400. doi:10.1093/mnras/sty3024

Neese, F., Wennmohs, F., Becker, U., and Riplinger, C. (2020). The ORCA Quantum Chemistry Program Package. J. Chem. Phys. 152, 224108. doi:10.1063/5.0004608

Norris, B. R. M., Tuthill, P. G., Ireland, M. J., Lacour, S., Zijlstra, A. A., Lykou, F., et al. (2012). A Close Halo of Large Transparent Grains Around Extreme Red Giant Stars. Nature 484, 220-222. doi:10.1038/nature10935

Perdew, J. P., Burke, K., and Ernzerhof, M. (1996). Generalized Gradient Approximation Made Simple. Phys. Rev. Lett. 77, 3865-3868. doi:10.1103/ physrevlett.77.3865

Tielens, A. G. G. M., and Hagen, W. (1982). Model Calculations of the Molecular Composition of Interstellar Grain Mantles. Astron. Astrophys. 114, 245

van Dishoeck, E. F., Herbst, E., and Neufeld, D. A. (2013). Interstellar Water Chemistry: From Laboratory to Observations. Chem. Rev. 113, 9043-9085. doi: $10.1021 / \mathrm{cr} 4003177$

Wales, D. J., and Doye, J. P. K. (1997). Global Optimization by BasinHopping and the Lowest Energy Structures of Lennard-Jones Clusters Containing up to 110 Atoms. J. Phys. Chem. A. 101, 5111-5116. doi:10. 1021/jp970984n

Watanabe, N., and Kouchi, A. (2008). Ice Surface Reactions: A Key to Chemical Evolution in Space. Prog. Surf. Sci. 83, 439-489. doi:10.1016/j.progsurf.2008. 10.001 
Watson, R. A., Rebolo, R., Rubiño-Martín, J. A., Hildebrandt, S., Gutiérrez, C. M., Fernández-Cerezo, S., et al. (2005). Detection of Anomalous Microwave Emission in the Perseus Molecular Cloud with the COSMOSOMAS Experiment. ApJ 624, L89-L92. doi:10.1086/430519

Weigend, F., and Ahlrichs, R. (2005). Balanced Basis Sets of Split Valence, Triple Zeta Valence and Quadruple Zeta Valence Quality for $\mathrm{H}$ to Rn: Design and Assessment of Accuracy. Phys. Chem. Chem. Phys. 7, 3297. doi:10.1039/ b508541a

Zeller, E. J., Ronca, L. B., and Levy, P. W. (1966). Proton-induced Hydroxyl Formation on the Lunar Surface. J. Geophys. Res. 71, 4855-4860. doi:10.1029/ jz071i020p04855
Conflict of Interest: The authors declare that the research was conducted in the absence of any commercial or financial relationships that could be construed as a potential conflict of interest.

Copyright (๑ 2021 Mariñoso Guiu, Ferrero, Macià Escatllar, Rimola and Bromley. This is an open-access article distributed under the terms of the Creative Commons Attribution License (CC BY). The use, distribution or reproduction in other forums is permitted, provided the original author(s) and the copyright owner(s) are credited and that the original publication in this journal is cited, in accordance with accepted academic practice. No use, distribution or reproduction is permitted which does not comply with these terms. 